\title{
Comparison between Two Genera, Species and Cultivars in Lactuceae IV. Effects of seed-irradiation on mitosis
}

\author{
M. Z. Haque and M. B. E. Godward \\ School of Biological Sciences, Queen Mary College, London EI 4NS, U.K.
} Accepted February 27, 1985

Previous work (Ahmad 1978, Ahmad and Godward 1981, Al-Allaf 1976, Al-Allaf and Godward 1979, Al-Rubeai and Godward 1981, 1982) has shown that there are considerable differences in sensitivity to radiation between closely related plants, not only between genera of the same family but between species of the same genus (Al-Rubeai 1981) and even between cultivars of the same species. The attribution of these differences in sensitivity to differences in DNA amount (Sparrow and Miksche 1961) cannot reasonably account for the differences between cultivars where the DNA amounts are nearly, if not totally, identical. A recent investigation (Ahmad 1978) on Cicer arietinum, was not entirely conclusive. There has been no such investigation in the Lactucoideae section of Compositae; the present study of three cultivars of Lactuca sativa, one wild species of Lactuca ( $L$. serriola) and the presumed ancestor of $L$. sativa, was therefore undertaken.

Ahmad (1978) had shown that those varieties which were more sensitive to radiation, assessed by growth-inhibition studies, also showed more chromosome aberrations at the same dose than the less sensitive varieties. The importance of this observation is clearly increased, the more such cases are demonstrated, and if no exceptions to it are found. Chromosome aberrations at the same dose were therefore scored in the above-mentioned varieties and species.

Relatively few investigations have compared the sensitivity to radiation of species which are putatively ancestral or descendant, closely or distantly related, and the cultivars derived from them. The wild Lactuca serriola is regarded as very close to, and possibly ancestral to the now doubtfully extant wild Lactuca sativa from which the cultivars Tom Thumb, Webbs Wonderful and Green Cos are derived.

\section{Materials and methods}

a) Control material; determination of germination time and time of first division of dormant seeds under standard condtions

To know the exact duration of time required for seed germination following soaking and for the occurrence of the first mitotic division using dormant seeds under standard conditions, the following procedures were carried out in a preliminary investigation, on three cultivars (Tom Thumb, Webb's Wonderful and Lobjoit's Green Cos) of Lactuca sativa L., L. serriola L. and Cichorium intybus L., (Witloof). 


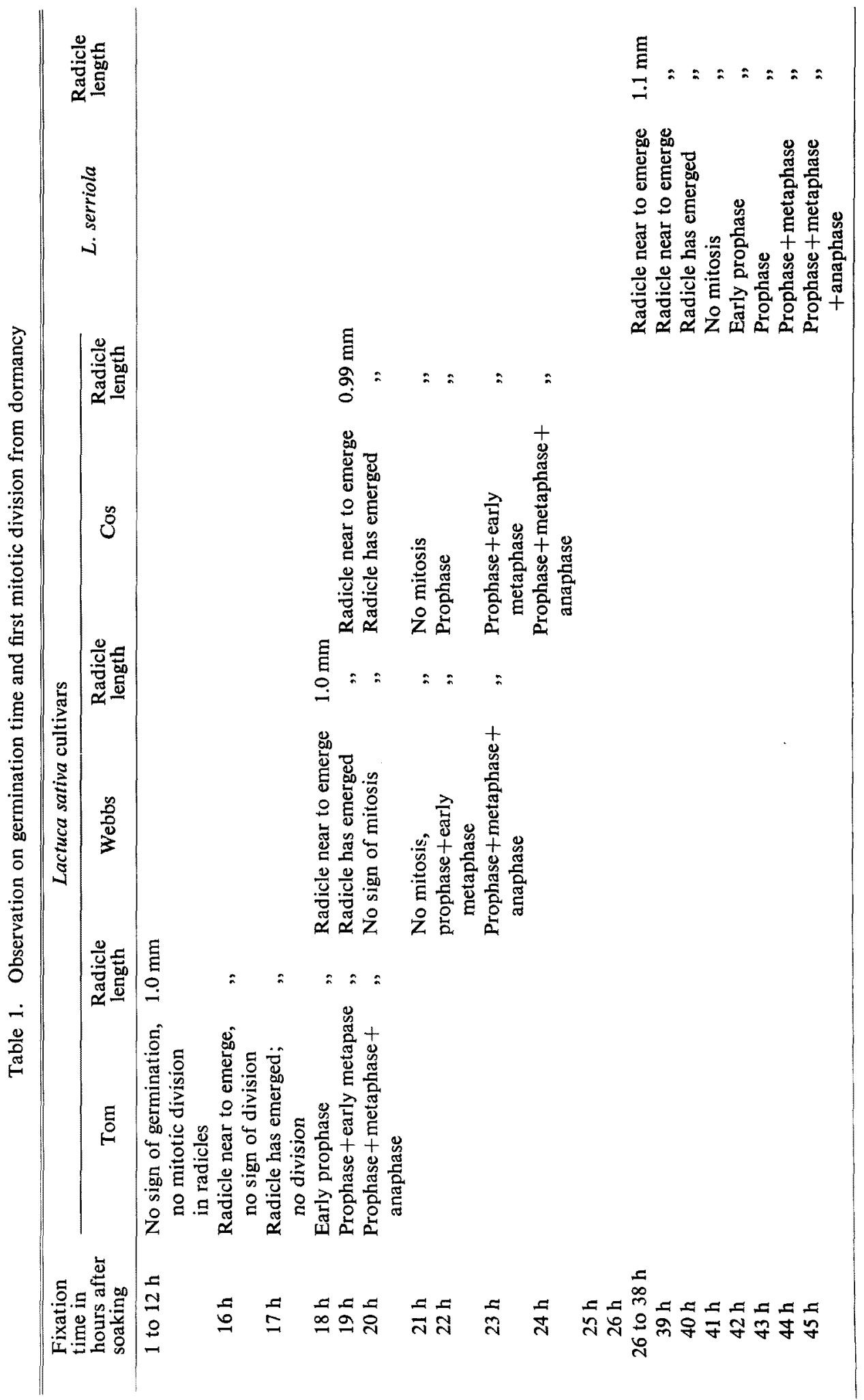


i. The four-months old dry dormant seeds were kept in a desiccator for about three months until no further loss in weight occurred.

ii. The seeds were then placed on filter paper in sterile petri dishes together with $3.5 \mathrm{ml}$ of sterile distilled water at $19-23^{\circ} \mathrm{C}$, in subdued light beneath ordinary white paper without special control of illumination.

iii. In order to know the exact time when the cells in the radicle begin to divide, the seeds were fixed in 1:3 acetic alcohol at hourly intervals after contact with water.

The radicles hourly fixed even before germination, were hydrolysed in $1 \mathrm{~N} \mathrm{HCl}$ for 10 minutes at $60^{\circ} \mathrm{C}$ and stained in Feulgen's solution for 2 hour. The seed coats were opened, the radicle removed and squashed in a drop of acetocarmine.

In each case 10 radicles fixed at the same time were squashed separately and examined initially for presence or absence of prophase.

b) Irradiated material with controls

A fresh set of controls were investigated at the same time as the seed-irradiated material. Irradiation was in a ${ }^{60} \mathrm{Co}$ unit.

i) Mitotic index

This was investigated in two cultivars (Tom Thumb and Cos) of L. sativa and in L. serriola.

Non-irradiated seeds and seeds given exposures of 10, 20 and $30 \mathrm{Kr}$ were germinated in sterile petri dishes on moist filter paper, under standard conditions as previously described. In addition exposures of 50,60 and $80 \mathrm{Kr}$ were also given, but no mitotic index determinations were made at these doses. Root tips from controls and treated samples of the two cultivars were fixed, in 1:3 acetic-alcohol at $24,48,72$ and 96 hours. Feulgen squashes were made from root tips as previously described. Each plant was scored separately, the sample size being 10 plants (each represented by its primary root tip meristem) of each control and in each dose. From each root tip, all cells in each of 10 randomly selected fields of view, as well as the number of dividing cells, i.e. number of cells in prophase, metaphase, anaphase and telophase were counted. In each root tip from 500-632 cells were scored; thus in total, 5000-6320 cells were scored in each control and in each treated sample. These methods permit the construction of frequency histograms.

ii) Estimation of anaphases and telophases with bridges

Anaphase and telophase bridge formation at each dose and in controls were scored as a measure of sensitivity to radiation, in which the two cultivars (Tom and Cos) of $L$. sativa were compared with $L$. serriola. Figs. 2-7 illustrate some of the cytological aberrations found.

The numbers of anaphases and telophases with and without bridges were scored. The numbers of anaphases and telophases with bridges are represented separately as the percentage of the total anaphases or telophases per root tip.

Scoring was 24 hours after soaking in all except $L$. serriola, when it was 48 hours after soaking, these being the times of first anaphases from dormancy (Table 1). 


\section{Results}

a) Control material

i) Determination of germination time

Under standard conditions, the duration of time prior to seed germination (i.e. testa burst) in different cultivars and species was as follows: (see Table 1)
L. sativa cultivars: Tom
17 hours from soaking

Webbs

19 hours from soaking

L. serriola

Cos 20 hours from soaking 40 hours from soaking

ii. Time of first division from dormancy

No mitotic divisions were found prior to testa burst. Observations on mitosis are represented in Table 1. Rapid germination was a feature of all the control materials although there is some little difference in germination times of the cul-

$$
\text { FREQUENGY DISTRIBUTION OF MITOTIC INDEX }
$$

(CONTROL ano IRradiated SeEdS) at 24 or 96 hOURS after soaking (CF. TABle 1)

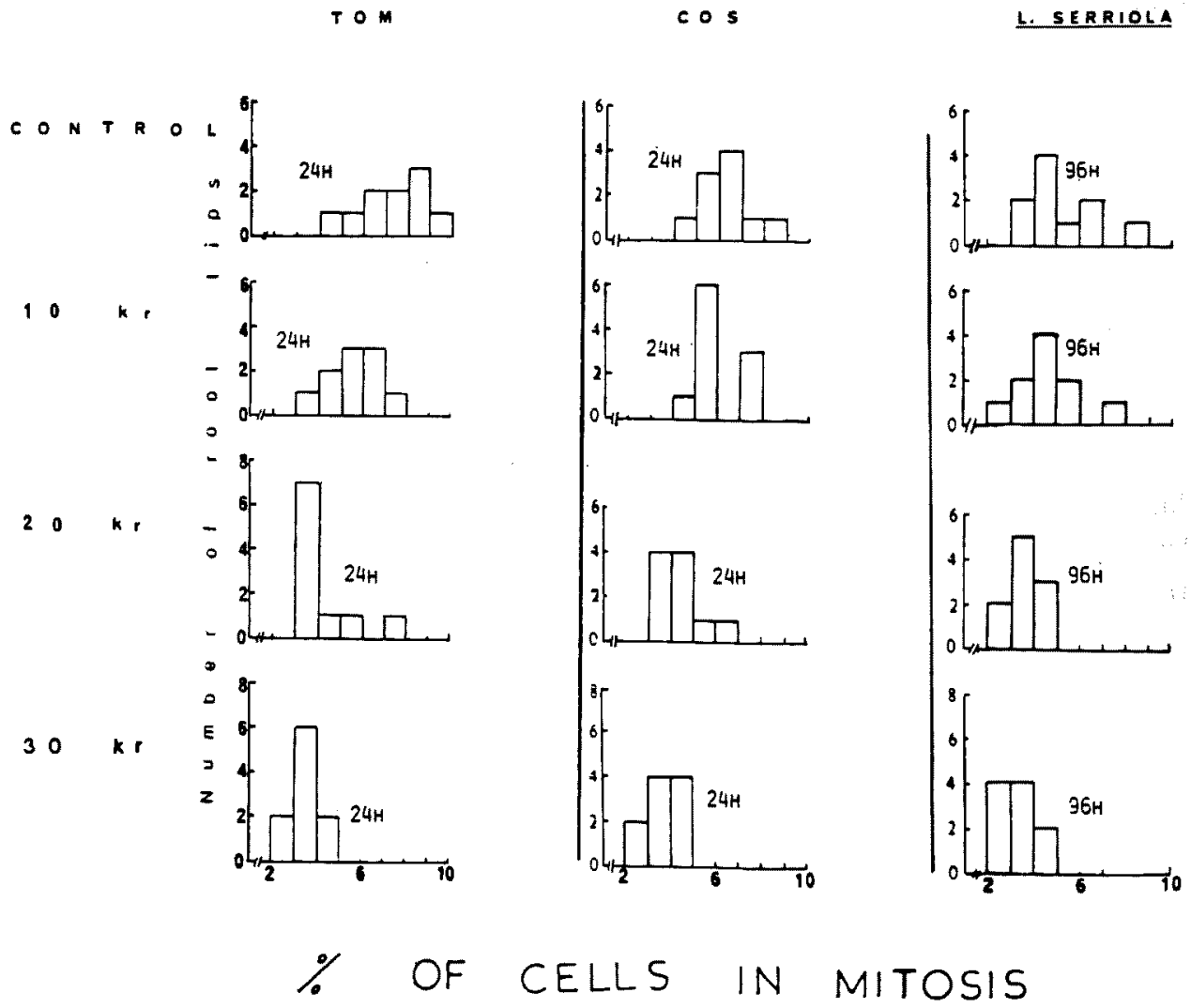

Fig. 1. First anaphase in controls was scored earlier than in the irradiated material, when delay increased with dose: from 22 to 24 hours in Tom and Cos, and from 48 to 72 hours in L. serriola. 

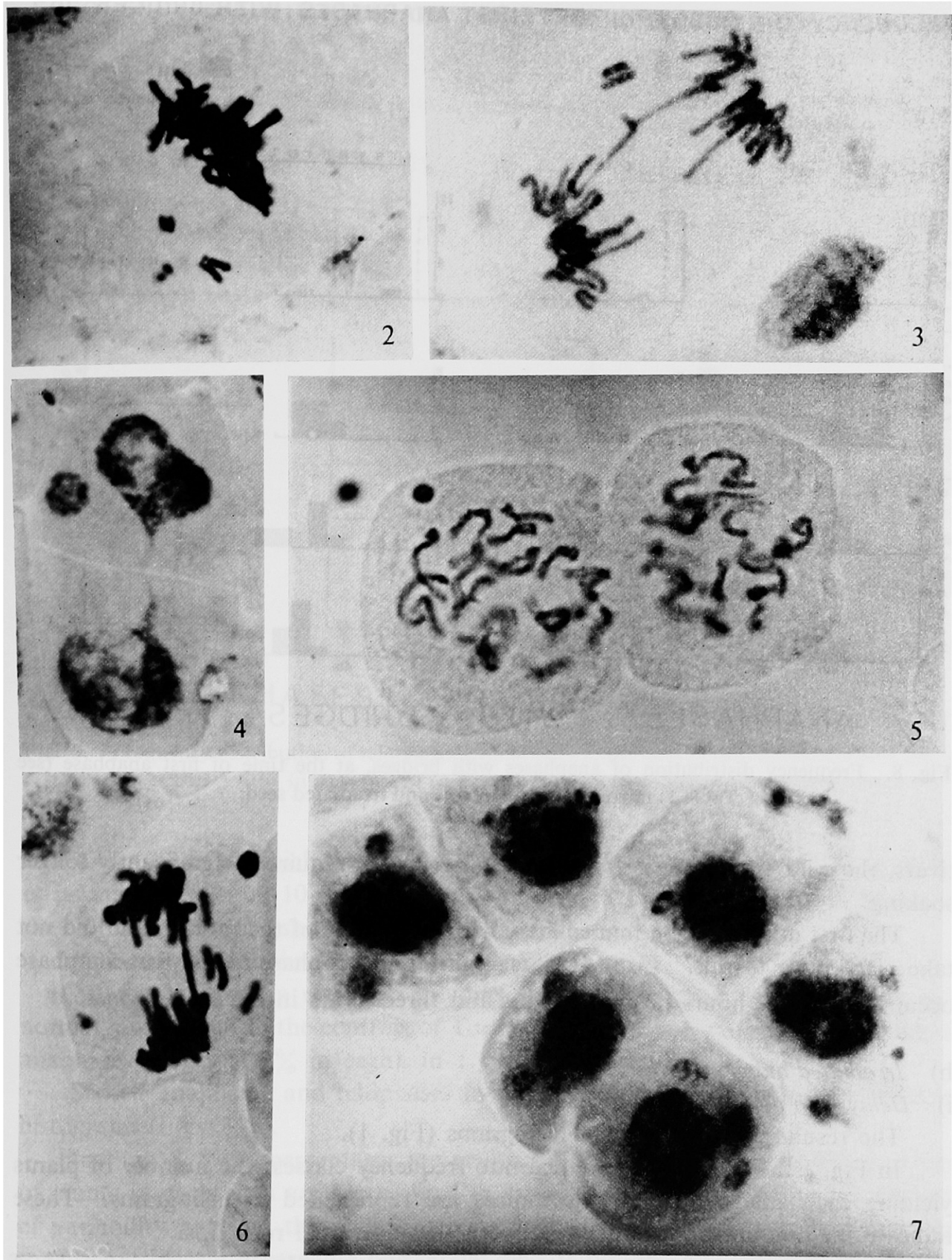

Figs. 2-7. 2, L. serriola, $30 \mathrm{Kr}$. First cell division in root tip cell. Fragments, consisting of paired sister chromatids, resulting from chromosome breakage in Gl. $(\times 1760) 3$, L. serriola, $30 \mathrm{Kr}$. First division from dormancy in root tip cell. Anaphase with a single bridge. Fragments, consisting of paired sister chromatids, resulting from chromosome breakage in Gl. $(\times 1760) \quad 4, L$. sativa cultivar Webbs, $30 \mathrm{Kr}$. First division from dormancy in root tip cell. Telophase with a persistent bridge and a single micronucleus. $(\times 1760) 5$, Tom, $30 \mathrm{Kr}$. 2nd or later division in root tip cell. Prophase with a single micronucleus. $(\times 1760) 6$, Tom, $30 \mathrm{Kr}$. Anaphase with a single bridge, lagging fragments and a micronucleus. $(\times 1760) \quad 7$, Tom, $30 \mathrm{Kr}$. Interphase, showing persistent accumulated micronuclei from preceding divisions. $(\times 1760)$. 
FREQUENCY DISTRIBUTION OF FIRST ANAPHASES WITH BRIDGES

T $\mathbf{~ M ~}$

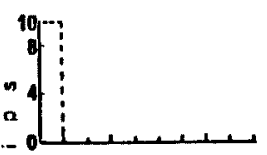

$+$
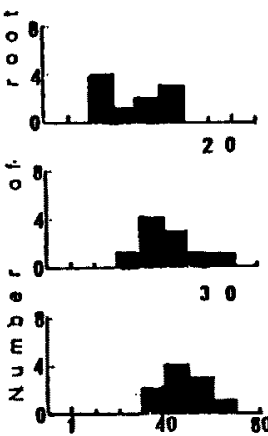

$\%$ ANAPHASES c o s
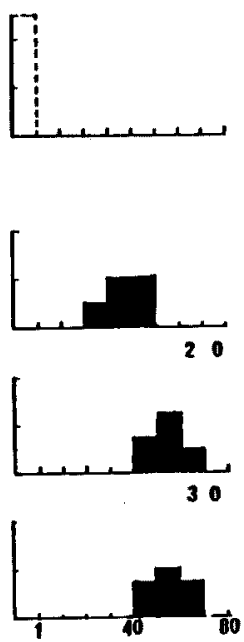

WITH
Wilh brldges

- I-without "

L. SERAIOLA
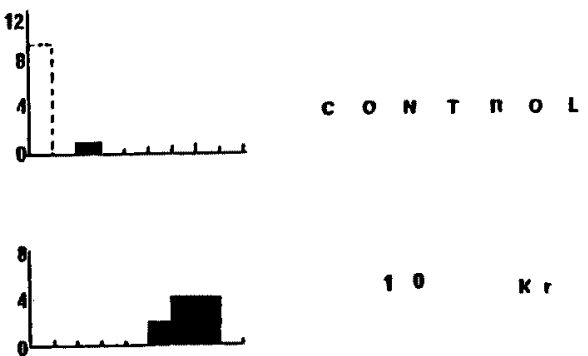

$10 \quad k r$

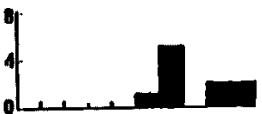

20

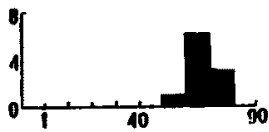

30

BRIDGES

Fig. 8. Frequency distribution of anaphases with bridges, at the time of first anaphase (see Table 1) in root tips of controls and irradiated seed.

tivars, the wild species $L$. serriola is the only one to require a significantly longer soaking.

The first mitosis began immediately with emergence of the radicle, and did not take place prior to this. From the first sighting of prophase to the first anaphase occupied $1 \frac{1}{2}$ to 2 hours in the cultivars and three hours in Lactuca serriola.

b) Irradiated material with controls

i) Delay with dose and mitotic index

The results are presented in histograms (Fig. 1).

In Fig. 1 the indices are grouped into frequency classes; the number of plants yielding each category of mitotic indices are represented in histograms. These indicate the amount of variability between the individual root tips.

The percentages of cells in mitosis represent the pooled scores for 10 field of view from each of 10 plants of each cultivar or species. In the two cultivars Tom and Cos, there was little delay in mitosis at the low doses of 10,20 and $30 \mathrm{Kr}$. There was delay at the higher doses of 60 and $80 \mathrm{Kr}$ and these doses were subsequently lethal. There was delay in mitosis in L. serriola, at the low doses of 20 and $30 \mathrm{Kr}$. This delay amounted to approximately 15 hours. The higher doses of 60 and 80 $\mathrm{Kr}$ produced further delay and these were again subsequently lethal..

Thus $L$. serriola shows its greater sensitivity in the delay of mitosis at 10,20 


\section{FREQUENCY DISTRIBUTION OF FIRST TELOPHASES WITH BRIDGES}

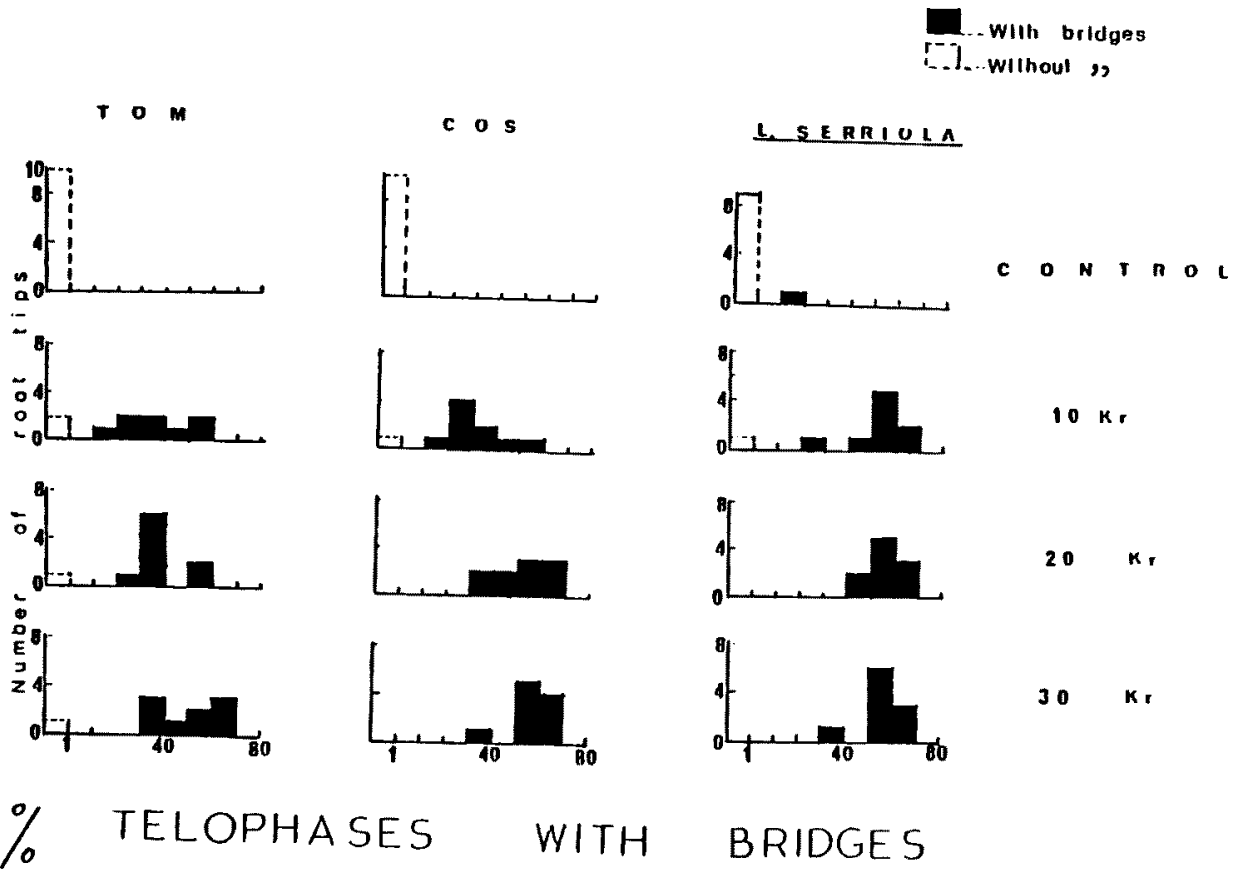

Fig. 9. Frequency distribution of telophases with bridges at the time of first telophase (see Table 1) in root tips of controls and irradiated seed.

and $30 \mathrm{Kr}$, doses at which there was little delay in the cultivars; and in the lowering of its mitotic index at $10 \mathrm{Kr}$ without appreciable subsequent recovery as occurs in the cultivars (data not shown).

ii) Estimation of anaphases and telophases with bridges

Mitosis in root tip meristem in controls of each cultivar and $L$. serriola was normal, except that in the controls of Tom and $L$. serriola, a very low percentage of anaphase bridges (1.7\% in each), in 1 out of 10 root tips was scored.

Scored anaphases and telophases in the irradiated materials are presented in histograms (Figs. 8, 9).

In Figs. 8, 9 the percentages are grouped into frequency classes; the numbers of plants in each class are represented in histograms. These indicate the amount of variability between the individual root tips. Although the variation between root tips is clearly observable, the modal class is different at each dose in the two cultivars and $L$. serriola. The percentages of cells with bridges increase with increase in dose, and are highest for $L$. serriola. Also the range of frequency classes for two cultivars Tom and Cos at all doses and for Cos beyond those for Tom (Figs. 8, 9). Thus the more radio-sensitive cultivars or species have more aberrations than the resistant. 


\section{Discussion}

The order of relative sensitivity shown by aberrations/dose is as follows:

$\left.\begin{array}{ll}\begin{array}{l}\text { most resistant } \\ \text { less resistant }\end{array} & \text { Tom } \\ \text { least resistant (sensitive) } & \text { Cos }\end{array}\right\} \begin{aligned} & \text { L. sativa } \\ & \text { L. serriola }\end{aligned}$

The relative dose response of the Lactuca cultivars and species studied as shown by the amount of aberration formation is exactly as shown independently by growth inhibition studies (not presented).

From the results of the present work it could be concluded that the chromosomes of $L$. serriola are actually more likely to be broken and rejoined so as to give bridges than those of the two cultivars $L$. sativa, Tom and Cos. Those of Cos are slightly more likely to be broken than those of Tom. Longer repairing times may be the cause of increased mitotic delay. Thus the wild species appears more sensitive than the cultivars. In Cicer arietinum also (Ahmad and Godward 1981) the more radiation-sensitive cultivar showed more chromosome aberrations at the same dose, than the more resistant cultivar.

Consideration of the possible relationship to DNA amounts, nuclear volume, chromosome morphology and taxonomy will be published in a later paper.

\section{Summary}

The germination time under standard conditions was determined in control material of three cultivars of Lactuca sativa and L. serriola, and also the time of first nuclear division from dormancy, together with mitotic index at stated times from onset of mitosis. The effect of seed irradiation on the mitotic index and on the production of anaphase bridges at the same dose in the different cultivars or species was investigated. Results are presented as histograms and/or tables. The order of sensitivity to radiation of two of the cultivars of $L$. sativa and $L$. serriola, determined by growth inhibition studies, not presented, was as follows:
most resistant
less resistant
$\left.\begin{array}{l}\text { Tom Thumb } \\ \text { Cos }\end{array}\right\}$
L. sativa
least resistant (most sensitive)
L. serriola

More aberrations were produced at the same dose in the sensitive species. The results are discussed.

\section{Bibliography}

Ahmad, S. 1978. Radiation studies in cultivars of Cicer arietinum, Ph. D. Thesis, University of London.

- and Godward, M. B. E. 1981a. Comparison of radio-resistant with a radiosensitive cultivar of Cicer arietinum L. I. Pinpointing the first anaphase from dormancy in untreated material. Environ. Exp. Bot. 21: 135-142.

- and - 1981b. Comparison of a radioresistant with a radiosensitive cultivar of Cicer arietinum L. II. Differences in the number of chromosome aberrations at the same dose. Environ. Exp. Bot. 21 : 143-151. 
Al-Allaf, S. M. 1976. Radiation studies in Helianthus annuus. Ph. D. Thesis, University of London. - and Godward, M. B. E. 1979. Effect of radiation on chiasma frequency in sunflower (Helianthus annuus). Cytologia 44: 821-833.

Al-Rubeai, M. A. F. 1981. Radiosensitivity of dormant Phaseolus seeds. Environ. Exp. Bot. 21: 71-74.

- and Godward, M. B. E. 1981. Genetic control of radiosensitivity in Phaseolus vulgaris L. Environ. Expt. Bot. 21: 211-216.

- and - 1982. Effects of acute gamma irradiation of four varieties of French beans. Genetica Iberica 34: 83-100.

Sparrow, A. H. and Miksche, J. P. 1961. Correlation on nuclear volume and DNA content with higher plant tolerance to chronic radiation. Science 134: 282-283. 